\title{
All Bids for One and One Does for All: Market-Driven Multi-Agent Collaboration in Robot Soccer Domain
}

\author{
Hatice Köse, Çetin Meriçli, Kemal Kaplan and H. Levent Akın \\ Boğaziçi University \\ Department of Computer Engineering \\ 34342 Bebek, Istanbul, TURKEY \\ \{kose, cetin.mericli, kaplanke, akin\}@boun.edu.tr
}

\begin{abstract}
This work proposes a novel approach for introducing market-driven strategy to robot soccer domain in order to solve vital issues related to multiagent coordination. In robot soccer, two teams of robots compete with each other to win the match. For the benefit of the team, the robots should work collaboratively, whenever possible. Market-driven approach applies the basic properties of free market economy to a team of robots, to increase the profit of team as much as possible. This approach is based on the assumption that maximizing individual profit will approximate global profit maximization. In several works, this method was applied to some open issues in multi-agent systems like multi-robot exploration and coordination, but these implementations were limited. In this work, this approach is applied for the first time to the robot soccer domain, which is being a complex, dynamic and real-time event, one of the prominent topics of multi-agent research. In this paper, a market-driven collaborative task allocation algorithm for the robot soccer domain is proposed and experimental results are discussed.
\end{abstract}

Keywords: Market-driven, multi-agent, collaboration, robot soccer.

\section{Introduction}

The use of cooperative robotics is becoming more prominent in many key application areas. Multi-agent teams, in which agents cooperate with each other and/or with human beings, become popular as their performance are shown to be better, more reliable and more flexible than single robots, in a variety of tasks (see $[1,2]$ for many papers about cooperative robotics). Unfortunately there are yet limited number of applications in this area, and many of these are about toy problems or limited implementations of applicable problems due to the difficulty of the general problem. Problems in the coordination of the robots, efficient usage of limited resources and communication burden discourages researchers from working on real-time problems with dynamic environments. Robot soccer is such an environment, with its real-time, complex and dynamic nature, and implementation of cooperative robotics to legged robots makes it even more challenging where locomotion (moving the legged robots) becomes a real bottle-neck.

Robot soccer has many important problems, like team localization, planning, task allocation, and multi-robot coordination, which can not be solved trivially. Therefore 
there are limited number of applications in this area. In many of them robot coordination is not implemented since it is found costly for a real-time application, and the rest tend to use simple mechanisms that are far from getting the full benefit of collaborative team work. For example CMPack'02 quadrupled legged-robot (AIBO) team from Carnegie-Mellon University which competed in Robocup 2002 [3] uses a variant of bidding mechanism for dynamic task allocation. In their approach, each candidate calculates their fitness for different roles and the fittest one is assigned to that role. Also some works use multi-agent based perception for team localization; [4, 5] work on multi-robot localization, [4] used statistical methods to integrate local map information of one robot to the others, but this requires the detection of other robots, getting their local info, and integrating all to update one's own info. In cases where either shared info is not reliable or detection of others is not trivial, as in robot soccer, this method is hard to use. In [5] local information coming from individuals is integrated (robots only share their own location and the location of ball), but individual information is always more reliable, and individuals do not gain anything from sharing their information. Additionally since the shared information is not very reliable, this only increases the communication burden. Algorithms have been introduced for multi-robot perception and object (e.g. ball) tracking $[6,7,8]$. Another approach combines local maps constructed by Extended Kalman filter, and transform these to overall map in only one iteration $[9,10]$. A couple of works were done on single map construction. Multiagent map construction requires an optimum positioning of all the team members [11] to gather maximum amount of information about the environment to construct the best map about the environment [12].

In the market-driven approach, individuals look for their benefit and try to make as much profit as they could as in free market economy. Almost every resource can be traded (i.e. sensors, map info, tasks to be completed, etc). A task associated to a robot can be put on an auction and be sold to a robot which offered a lower price than the owner. Since decentralized implementation of this auction mechanism is possible (there is no superior being organizing auctions) and tasks of a robot may be re-associated to another robot which becomes more proper for that task in time, the market-driven approach is robust and free from single point failure (e.g. even when a robot is taken out of field by the referee in the quadrupled legged robot matches, the rest of the team can take the lost robot's task and continue to play).

The market-driven approach is used in $[13,14]$ for robot coordination. The work in [15] introduces the approach to multi-robot exploration. Implementations seem to be successful, but domains like agricultural areas are not dynamic and do not require fast task allocation, planning and coordination as in robot soccer, since the environment is almost static, and not complex as robot soccer.

This paper proposes a novel approach called "Collaboration by competition / cooperation" for the dynamic collaborative task allocation problem for multi-agent robotic soccer, based on free market driven method. Market driven methodology can be used to solve many open issues in robot soccer, and in this work, a solution to one of these issues is implemented. The method is based on the assumption that when the individuals work to increase their individual profit due to the benefit of the team, the profit of the team will be maximized. 
The organization of the paper is as follows: In the second section, the market-driven method is defined generally. The third section makes a brief introduction to robot soccer. In the fourth section detailed information about the proposed approach can be found. In the fifth section, results of the application of the proposed approach are presented. In the last section, conclusions and suggestions for future work are given.

\section{Market-Driven Method}

The idea of market-driven method for multi-robot teams is based on the interaction of the robots among themselves in a distributed fashion for trading work power - information and hence providing "Collaboration by competition-cooperation" as we call it. In general, there is an overall goal of the team (i.e., building the map of an unknown planet, harvesting an agricultural area, sweeping buried landmines in a particular area, etc.. ). Some entity outside of the team is assumed to offer a payoff for that goal. The overall goal of the system is decomposed into smaller tasks and an auction is performed for each of these tasks. In each auction, participant robots (who are able to communicate among themselves) calculate their estimated cost for accomplishing that task and offer a price to the auctioneer. At the end of the auction, the bidder with the lowest offered price will be given the right of execution of the task and receives its revenue on behalf of the auctioneer.

There are many different possible actions that can be taken. A robot may open another auction for selling a task that it won from another auction, two or more robots may cooperatively work and get a task which is hard to accomplish by a single robot, or, for a heterogeneous system, robots with different sensors/actuators may cooperate by resource sharing (for example, a small robot with a camera may guide a large robot without a vision system for carrying a heavy load). The main goal in the free-markets is maximizing the overall profit of the system. If each participant in the market tries to maximize its profit, as a result of this, overall profit for the system is expected to increase.

A cost function is defined for mapping a set of resources (required energy, required time, etc...) to a real number and the net profit is calculated by subtracting estimated cost for accomplishing the task from the revenue of the task. For example, in Eq.1 an estimated cost is calculated from the time required for the robot to cover the distance to the task, align itself according to the task, and clearance of range which is used to avoid objects on the path between the robot and the task, and suitable $\mu_{i}$ 's acting as weights to show the importance of each issue in the overall cost. Detailed descriptions of the parameters are given in Table 1.

$$
C_{e s}=\mu_{1} \cdot t_{d i s t}+\mu_{2} \cdot t_{\text {align }}
$$

The general structure of the process would be better understood by referring to the following scenario: Suppose there are two robots and two tasks in the environment. The costs of tasks calculated by the robots are as in Figure 1. Given the assumption that the goal is gaining the highest possible profit, both robots would choose the cheapest tasks for them individually, which seems to be the most profitable choice for them at 


\section{Task B}

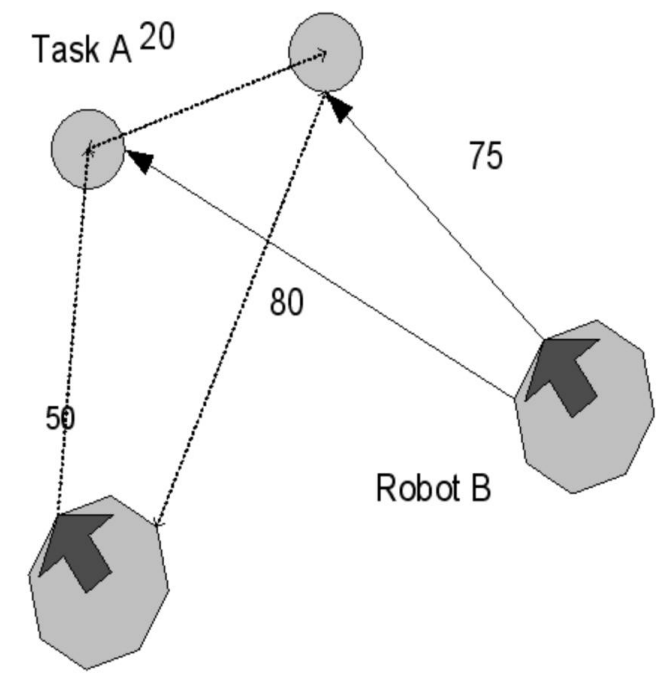

Robot A

Fig. 1. Market-driven scenario

first glance. So Robot A would take Task A and Robot B would take Task B. This would cost 50 for Robot A and 75 for Robot B, totally 125 for the team. Consider the case where beneath the individual profits, the team profit is important as well. Suppose Robot B calculates that if Robot A takes both tasks, this would cost the team only 70, so if the Robot B passes the execution right of the Task B to the Robot A, the completion cost of two tasks for the team would be a total of 70 . Therefore, the overall team profit would be increased.

\section{Robot-Soccer Domain}

In robot soccer, teams of robots, that are capable of seeing and moving play matches against each other, and the team with highest goal score wins the match like in real soccer. In order to do this, the player robots must detect their location, the goals, the ball, the members of their team and the opponent team members (optional for high level planning), and place the ball in the opponent team's goal to score. A robot is typically expected to find its own location using the landmarks (artificial or natural) in the field, and then use this information to find the location of the ball and goal. So localization is a vital problem for robot soccer. Image processing, planning and locomotion are the other important problems. 

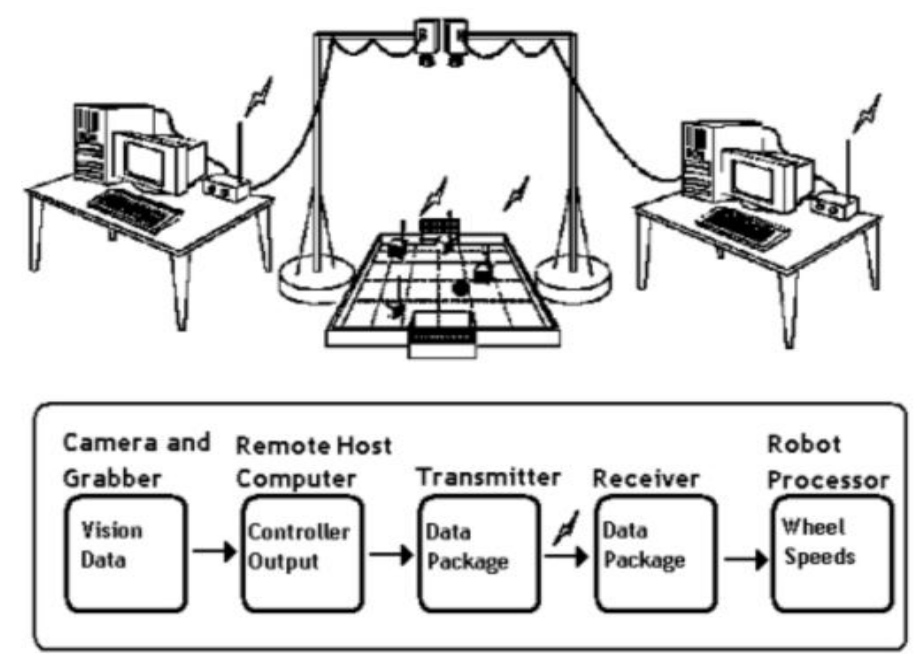

Fig. 2. The MIROSOT environment

Federation of International Robot-soccer Association (FIRA) is an association for international robot soccer [16] where wheeled and legged robots compete in the official games. MIROSOT is one of the categories for these robots. The MIROSOT competitions involve researching and designing a robot soccer team, which will meet the requirements of MIROSOT game rules. Since the environment is very dynamic, a complex controller design is required to perform sophisticated tasks like team organization.

The main part of the MIROSOT soccer system is the remote host computer where the controller is located (Figure 2). The controller processes the input from the vision system. The output of the controller is the communication data package. This package is sent to the robots through the RF module. The robots first process the package to extract the commands sent to them. In our case, the command only includes the left and right wheel velocity information. Then the robot transmits this information to its motor drivers. The work proposed in this paper is a part of the Robot Idman Yurdu soccer project [17].

In robot soccer, teams of robots, that are capable of seeing and moving play matches against each other, and the team with highest goal score wins the match like in real soccer. In order to do this, the player robots must detect their location, the goals, the ball, the members of their team and the opponent team members (optional for high level planning), and place the ball in the opponent team's goal to score. A robot is typically expected to find its own location using the landmarks (artificial or natural) in the field, and then use this information to find the location of the ball and goal. So localization is a vital problem for robot soccer. Image processing, planning and locomotion are the other important problems. 


\section{Proposed Approach}

The market-driven approach can be applied to several open issues in robot soccer. Dynamic task allocation is one of these issues. The following section states a market-driven dynamic task allocation algorithm for robotic soccer.

\subsection{Dynamic task allocation between team members}

There is a pool of predefined roles and there is a set of possible tasks for each role. These roles are primary attacker, secondary attacker, tertiary attacker and defender. There are two tasks for the roles: shoot or pass to a team member. Initially, each team member calculates its costs, including the cost of moving, aligning itself suitably for the task, and cost of object avoidance. Calculated costs are shared among the team members. After sorting the costs, each team member gets the appropriate role from the pool according to their rank in the ordered costs list (e.g. the member with the lowest defense cost becomes defender whereas the member with the lowest attack cost becomes primary attacker).Each role is assigned to exactly one team member. After the initial assignment, each member looks for another team member who can do this task for less cost by opening an auction on that task. If any robot can do this task with a lower cost, the task is assigned to that robot; therefore, the cost is decreased. The remaining robots behave according to their assigned roles, i.e. defender tries to locate itself on the middle of the line in between the own goal line and the ball. Since each robot has the cost information of all members of the team and the role assignment rules only depends on these cost values, each robot can decide that for which task it is most suitable. In other words, this process can be called as "Role Selection" instead of "Role Assignment". If one robot failed to accomplish its task required by its current role, the task remains uncompleted and possibly taken by another robot in the next auction.

The most important task is scoring the goal (making the ball enter the opponent's goal) in the robot soccer. So the ball is the most important object to be tracked, and should be moved to the opponent's goal successfully while avoiding the opponent team to scoring, so the ball should be in the control of the team members as much as possible. To provide the control of the ball and avoid opponent team to score as much as possible, all individuals in the team are encouraged to get the ball, and shoot it towards opponent goal. Consequently, the one who is closer to ball tries to get the ball to avoid opponents having the ball, and to shoot it to opponent goal. But if there are any other robots between the robot who owns the ball and opponent goal, a shoot would not be successful. In such a case, it might be more appropriate for that robot not to take the risk and pass the ball to another team member who can score goal. The robots calculate their defense cost in the cost calculation phase. After the primary attacker is assigned according to the attacking costs, the one with the lowest defense cost is assigned as defender, and it tries to take the best position between the ball and its own goal area to avoid any possible opponent team scores. The rest of the team are assigned their roles such that; in order of cost values, the robot with second good cost would serve as secondary attacker which would help the primary attacker in case of failure of a shoot, or completing an attack by having the ball after the ball is bounced from some obstacle on its way to the goal area. So it would place itself symmetric to the position of the ball to meet with the ball easily 
if it bounces back, the remaining robot is assigned as the third attacker, it tries to go to the ball, to help the other attackers incase of any failure.
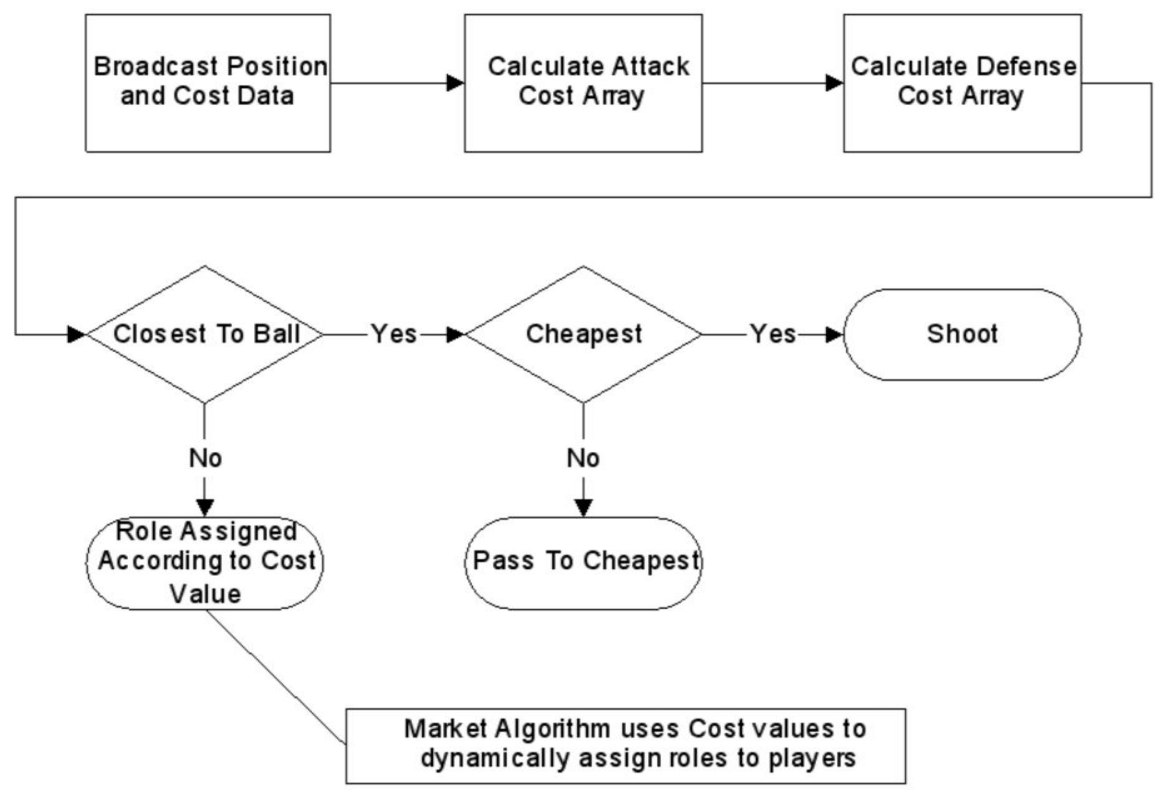

Fig. 3. Flow chart for task assignment

The detailed implementation of the approach is described with a flow chart in Figure 3. The cost functions used in the implementations are as follows:

$$
\begin{aligned}
C_{E S} & =\mu_{1} \cdot t_{\text {dist }}+\mu_{2} \cdot t_{\text {align }}+\mu_{2} \cdot \text { clear }_{\text {goal }} \\
C_{\text {bidder }} & =C_{E S}+\mu_{4} \cdot \text { clear }_{\text {ball }} \\
C_{\text {auctioneer }} & =C_{E S} \\
C_{\text {defender }} & =\mu_{5} \cdot t_{\text {dist }}+\mu_{6} \cdot t_{\text {align }}+\mu_{7} . \text { clear }_{\text {defense }}
\end{aligned}
$$

In Table 1, the parameters of the cost functions are described in detail. The $\mu_{i}$ 's in the cost functions are determined according to the to game policy, e,g, if a small value is selected as the $\mu_{1}$ (coefficient of distance to the opponent goal), the distance to the opponent goal does not have a significant contribution to the cost. As a result of this, the robot will decide to shoot regardless of the distance to the opponent goal area, hence, behave in a more defensive manner (the main purpose will become not to score but to keep the ball away from the own goal area). Miscalculation of a cost may lead the team to a wrong role allocation; therefore, the team members may behave unsuitably 
Table 1. Detailed description of parameters in the cost functions

\begin{tabular}{|l|l|}
\hline Term & Description \\
\hline$C_{E S}$ & Estimated score cost \\
\hline$t_{\text {dist }}$ & Time required to move for specified distance \\
\hline$t_{\text {align }}$ & Time required to align for specified amount \\
\hline$\mu_{i}$ & $\begin{array}{l}\text { Weights of several parameters to mention their relative importance } \\
\text { in the total cost function }\end{array}$ \\
\hline clear $_{\text {goal }}$ & Clearance from the robot to goal area-for object avoidance \\
\hline clear $_{\text {ball }}$ & Clearance from the robot to ball-for object avoidance \\
\hline clear defense & $\begin{array}{l}\text { Clearance from the robot to the middle point on the line between } \\
\text { middle point of own goal and the ball-for object avoidance }\end{array}$ \\
\hline$C_{\text {bidder }}$ & Estimated cost for bidder \\
\hline$C_{\text {auctioneer }}$ & Estimated cost for auctioneer \\
\hline$C_{\text {defender }}$ & Estimated cost for defender \\
\hline
\end{tabular}

for their situations until the next update is performed. The overall affect of this wrong task allocation on the team depends on the length of the update period. A long period may cause the team members to behave inappropriately to their situations for a long time but on the other hand, a short period of update may cause oscillation in the role assignment when two or more team members have close cost values (Due to sensory errors, the costs ripple over time and team members exchange roles too fast). This may lead the team to be stuck.

\section{Tests and Results}

The proposed approach in Figure 3 is implemented on Teambots, which is a multi-robot simulator designed for multi-agent applications [18]. It is used to simulate multi-agent behavior of MIROSOT teams, since their multi-agent structure is similar, and allows the user to do modifications when necessary. Notice that while keeping the general marketdriven idea, various kinds of different game strategies, tasks and scenarios can be developed. In this work, one of these is implemented to show the power of the method. For locomotion, a potential field based method which is trained by genetic algorithms is used to get a smooth movement. The 'MarketTeam' (team of robots controlled by market-driven strategy) played against the three teams described below. 30 matches were played against each team, and results are displayed in Table 2. There were six metrics measured during the experiments. Average Distance Ratio is the ratio of the sum of the distances of opponent players to the ball to the sum of the distances of our players to the ball. For the next three metrics, the game field is divided into three sections as in the Figure 4.

In the Figure $4, \mathrm{R}$ is the radius of a robot. The remaining two metrics are average of our scores and average of opponent scores. Brief descriptions of the opponent teams 


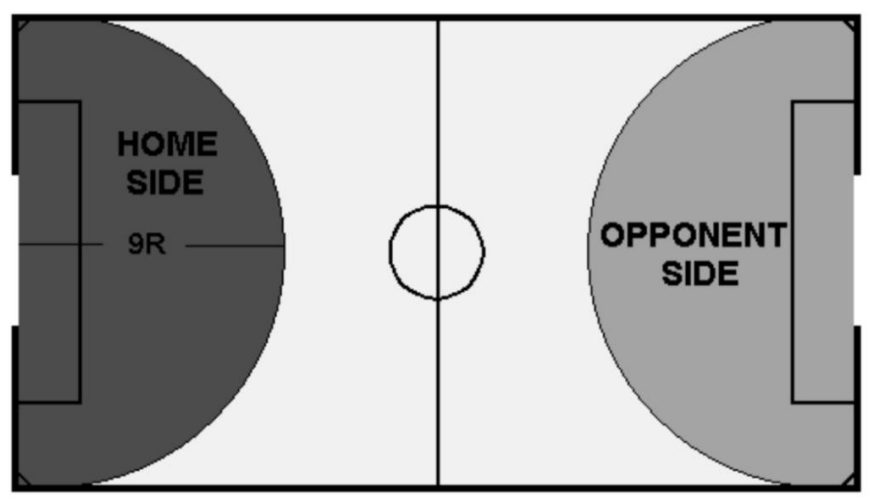

Fig. 4. Division of the field into three sections

are as follows: RIYTeam has a simple bidding mechanism based on the distance to ball, for task allocation, and used a potential field based method which is trained by genetic algorithms as MarketTeam to move after tasks are allocated [19]. AIKHomoG uses dynamic role assignment for strategy and potential fields for movement. Kechze uses dynamic role assignment for strategy and geometric calculations as the assignment criteria.

Table 2. Experiment results

\begin{tabular}{|l|l|l|l|l|l|l|}
\hline Team & $\begin{array}{l}\text { Avg. } \\
\text { Dist Ratio }\end{array}$ & $\begin{array}{l}\text { Ball is } \\
\text { on our side }(\%)\end{array}$ & $\begin{array}{l}\text { Ball is } \\
\text { on center }(\%)\end{array}$ & $\begin{array}{l}\text { Ball is } \\
\text { on opp. side }(\%)\end{array}$ & $\begin{array}{l}\text { Avg. } \\
\text { Our Score }\end{array}$ & $\begin{array}{l}\text { Opp. } \\
\text { Avg. Score }\end{array}$ \\
\hline AIKHomoG & 0,77 & 21,09 & 49,29 & 29,62 & 1,20 & 0,10 \\
\hline RIYTeam & 1,53 & 20,08 & 52,92 & 26,99 & 0,77 & 0,10 \\
\hline Kechze & 1,13 & 21,55 & 51,32 & 27,13 & 1,43 & 0,23 \\
\hline
\end{tabular}

In the test matches, It is seen in the results that the MarketTeam is succeeded to keep the ball away from its own goal area more than the opponent teams. As a result of this, our average score is considerably higher than opponent average score against all the three opponents. The game plan would change simply by changing the cost functions in order to define relative importance of defensive behavior over offensive behavior, and this gives a great flexibility in planning for us, which we lack with current algorithms. In Figure 5 the winner decides that it can not shoot the ball to goal since its front view is full of opponent defenders, then it passes the ball to the team member at best position. In Figure 6, the team member takes the ball from winner and shoots it to goal which 
will lead to a possible goal.

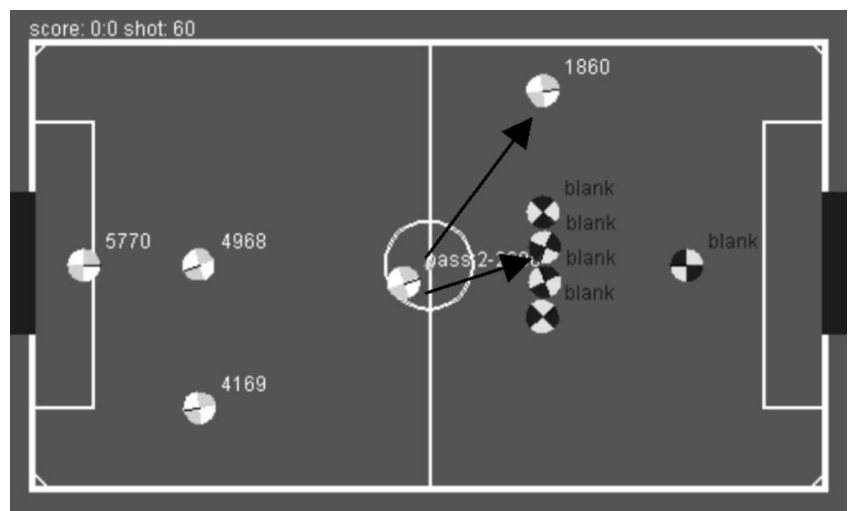

Fig. 5. Auctioneer passes the ball

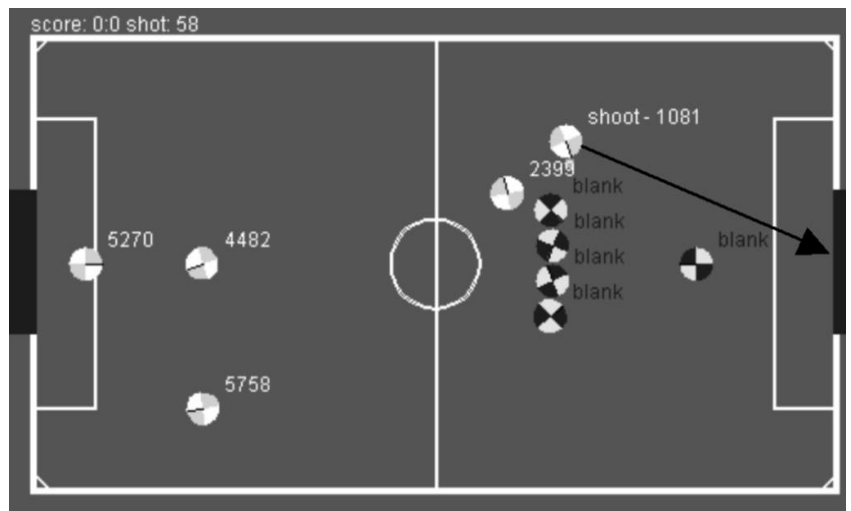

Fig. 6. The new winner shoots the ball to goal

\section{Conclusion}

The domain of multi-agent applications is a very challenging research area, which allows more robust, flexible, and faster solutions with less cost, more useful in areas such as industrial (e.g. agriculture), military applications (e.g. mine sweeping), planetary explorations, etc, where single robot or human solutions are either inefficient or 
the environment is hazardous for humans. Robot soccer is one of the new test beds for multi-agent domain, with its highly complex, dynamic and noisy nature bringing more limitations and complexity as being a real-time application. So, contrary to the domains with static environment with no real-time requirement, solutions for such a domain are not trivial, in general. The proposed approach is introduced to this domain for the first time in this work. The disadvantage that this limited communication might bring is that the system might converge on a suboptimal solution because of the entire team not communicating with each other. In the RoboCup soccer domain, there are only four players and one of them has a statically assigned role (the goal keeper), so the remaining three robots can easily keep in touch with each other. The distributed nature of the approach avoids single point failures of centralized approaches, and even if some of the robots are injured or lost the team continues to work, and the assigned goals of lost robots are handled and accomplished by the remaining robots, eventually. So system is highly robust. The market-based idea provides highly efficient resource sharing including the sharing of physical resources such as computational power and sensors, and informational resources such as a map of the environment, or global location of any mission-related object. Extending both physical and informational resources of the robot by trading with teammates allows robots to develop higher level plans and therefore, behave in a more intelligent way. So this would allow robots to take the full benefit of 'team spirit' which is the core idea of multi-agent research.

This mechanism gives a task to the most appropriate robot, which has the minimum estimated cost to accomplish that task, and force the robot to finish its task successfully. Otherwise, the robot is penalized by losing the job and paying off a penalty cost. This provides good behavior coordination in between the individual team members, and avoids possible conflicts, which are disastrous problems between team members (e.g. both team members compete for the ball, they block each other and consequently nobody could access the ball).

Besides the simplicity of cost calculation, communication and task allocation allow fast play which is a must for real-time games. With better alignment and shooting (with even a perfect plan, it is not trivial to make the robots do what they should, they can not align and shoot to the place they should perfectly, this is even worse in real robots so locomotion is a bottle-neck to test the algorithms) and new tasks to use for defenders, the success would be improved. The results show that system has high performance besides being fast and robust. Also dynamic task allocation due to the situation changes in the game makes the approach adaptive, robust and suitable to real-time events, where the team always has a plan B according to the situation; it becomes defensive or offensive according to the location of the ball and mode of the game.

When this implementation is carried to real physical world, the noise due to real world and limitations of physical devices might affect the sensors and cause miscalculations of cost functions so role changing between teammates which are in similar positions may be too fast and cause oscillations as described before. This would be effectively avoided by using suitable time stamps which forces the team members to keep their role at least for a limited duration of time.

The main possible disadvantage of market-driven task allocation for robotic soccer domain is the time requirement for the auctioning and utility calculation processes. 
Since some form of utility calculation is necessary in nearly all multi-agent task allocation problems, optimization of utility calculation is independent from market-driven approach. Time requirement constraints for auctioning might be satisfied by setting appropriate auction duration and time out for auctioneer values.

As a conclusion, there are still many open issues in multi-agent approach to robotic soccer domain and market-driven approach provides robust and efficient planning and resource sharing. As a future work, usage of the market-driven idea in multi-agent localization and perception would be done.

\section{Acknowledgement}

This project is supported by the Boğaziçi University Foundation and by Boğaziçi University Research Fund project 03A101D.

\section{References}

1. A. Saffiotti, "Proceedings Workshop WS7 Cooperative Robotics," IROS 2002, IEEE/RSJ International Conference on Intelligent Robots and Systems, September 30-October 4, 2002.

2. I. Ribeiro and A. Saffiotti (Eds), Lecture Notes from European Summer School on Cooperative Robotics, 2-7 September, 2002.

3. M. Veloso, S. Lenser, D. Vail, M. Roth, A. Stroupe, and S. Chernova, CMPack-02: CMU's Legged Robot Soccer Team, Carnegie Mellon University, Pittsburgh, October 2, 2002.

4. D. Fox, W. Burgard, H. Kruppa, and S. Thrun, A Monte Carlo Algorithm for Multi-Robot Localization, CMU-CS-99-120, March 1999.

5. A.W. Stroupe, T. Balch, "Collaborative Probabilistic Constraint Based Landmark Localization," Proceedings of the 2002 IEEE/RSJ Intl. Conference on Intelligent Robots and Systems, Page: 447-452, EPFL, Lausanne, Switzerland, October 2002.

6. M. Dietl, J.S. Gutmann and B. Nebel "Cooperative Sensing in Dynamic Environments," In Proc. of the IEEE/RSJ International Conference on Intelligent Robots and Systems (IROS 2001), Maui, Hawaii, 2001.

7. D. Schultz, W. Burgard, D. Fox and A.B. Cremers "Tracking Multiple Moving Objects with a Mobile Robot," In Proc. of the IEEE Computer Society Conference on Computer Vision and Pattern Recognition (CVPR 2001), Kauwai, Hawaii, 2001.

8. T. Schmitt, R. Hanek, S. Buck, and M. Beetz, "Cooperative Probabilistic State Estimation for Vision-based Autonomous Mobile Robots," In Proc. of the IEEE Intl. Conf. on Intelligent Robots and Systems (IROS 2001), Maui, Hawaii, 2001.

9. J. E. Guivant and E. M. Nebot, "Optimization of the Simultaneous Localization and MapBuilding Algorithm for Real-Time Implementation," IEEE Transactions on Robotics and Automation, Vol.17, No.3, Page: 242-257, June 2001.

10. G. Dissanayake, P. Newman, S. Clark, H.F. Durrant-Whyte, and M. Csorba, "A Solution to the Simultaneous Localization and Map Building (SLAM) Problem," IEEE Transactions on Robotics and Automation, Vol. 17, No. 3, June 2001.

11. A. W. Stroupe, Mission-Driven Collaborative Observation and Localization, Thesis Proposal, The Robotics Institute Carnegie Mellon University, December 2001.

12. J. Borenstein, H.R. Everett, and L. Feng, Navigating Mobile Robots, Systems and Techniques, A. K. Peters, Ltd., Wellesley, MA, 1996. 
13. M. B. Dias, and A. Stenz, A Free Market Architecture for Coordinating Multiple Robots, CMU-RI-TR-99-42, December 1999.

14. M. B. Dias, and A. Stenz, A Market Approach to Multi-robot Coordination, CMU-RI-TR01-26, August 2001.

15. R. Zlot, A. Stenz, M. B. Dias, and S. Thayer, "Multi-Robot Exploration Controlled by a Market Economy," Proceedings of the IEEE International Conference on Robotics and Automation, May 2002.

16. FIRA http://www.fira.net/, 2003.

17. Robot Idman Yurdu, http://robot.cmpe.boun.edu.tr/riy/riy.php, 2003.

18. T. Balch, "Teambots," http://www.cs.cmu.edu/ trb/TeamBots/Domains/SoccerBots, 2000.

19. K. Kaplan, Design and Implementation of fast controllers for Mobile Robots, Master Thesis, January 2003.

This article was processed using the $\mathrm{LT}_{\mathrm{E}} \mathrm{X}$ macro package with LLNCS style 